\title{
Bargmann Invariant and the Geometry of the Güoy Effect
}

\author{
R. Simon \\ The Institute of Mathematical Sciences, C.I.T. Campus, Madras 600 113, India \\ N. Mukunda \\ Centerfor Theoretical Studies and Department of Physics, Indian Institute of Science, Bangalore 560 012, India \\ and Jawaharlal Nehru Centre for Advanced Scientific Research, Bangalore 560012 , India
}

\begin{abstract}
We bring out the connection between Berry's geometric phase and a gauge invariant expression introduced by Bargmann. We exploit this relationship to show that the Güoy effect, which refers to the phase jump experienced by a focused beam as it crosses the caustic, is the geometric phase associated with the underlying Lobachevskian hyperbolic geometry.
\end{abstract}

Berry's discovery [1] of the geometric phase has led to much theoretical [2] and experimental [3-5] development. It has been realized that many earlier studies can be now viewed as forerunners of the Berry phase in special situations. The purpose of this Letter is twofold: First, to add to this list a gauge invariant phase introduced by Bargmann [6] and bring out its precise connection to the geometric phase; and second, to exploit this connection to unfold the geometry of the Giioy effect $[7,8]$. We show that the Giioy phase is the geometric phase associated with the metaplectic group.

The Giioy effect refers to the rapid phase change of amount $m \pi / 2$ suffered by a focused beam as it crosses the caustic; the dimension $m=\backslash$ for a focal line (cylindrical beam) and $m=2$ for a focal point (spherical beam). For convenience we consider Gaussian beams. They play a basic role in laser resonator systems and in guided beam optics [8]. In particular Giioy phases of Gaussian and Hermite-Gaussian beams directly enter the expression determining the resonant frequencies of laser modes [8].

There exists a fundamental similarity between the Schrödinger equation for a free particle of unit mass in two dimensions and the wave equation for a paraxial beam propagating along the $z$ direction in free space:

$$
i \underset{d z}{\partial \psi} \psi(x, y ; z)=-\frac{X}{2}\left(\begin{array}{cc}
d^{2}, & \partial^{2}- \\
\partial x^{2} & d y^{2}
\end{array}\right) \psi(x, y ; z)
$$

The $t$ evolution in quantum mechanics and the $\mathrm{z}$ evolution in optics are formally identical, with the role of $h$ in quantum mechanics played by $x=\lambda / 2 \pi$ ( $\lambda$ is the wavelength) in optics. In fact, wave optics can be obtained by formal quantization [9] of ray optics in exactly the same way as quantum mechanics from classical mechanics. Thus, the $z$ evolution of Gaussian laser beams and the $t$ evolution of Gaussian quantum wave packets are identical and, in particular, both suffer similar Giioy effects. Our analysis applies to both situations, and so we speak of $s$ evolution, with $s=z$ or $t$ as appropriate. Thus, given $\psi(x, y ; s)$ the Giioy phase $\varphi_{G}(s)$ is on the axis phase [8] $\arg \psi(0,0 ; s)$. We assume for brevity $\mathrm{ft}=\mathrm{X}=1$. Then in both cases the unitary $s$ evolution is generated by the constant Hamiltonian $\left(p_{x}^{2}+p_{y}^{2}\right) / 2$, where $p_{x}=-i \partial / \partial x$ and $p_{y}--i \partial / \partial y$.

In the course of his celebrated proof of Wigner's theorem on symmetry operators Bargmann [6] noted that given three states $\left|\psi_{0}\right\rangle,\left|\psi_{1}\right\rangle,\left|\psi_{2}\right\rangle$, while quantum mechanics allows a freedom in the choice of phase (gauge) for each state, the expression

$$
\varphi_{B}=\arg \left\langle\psi_{0} \mid \psi_{1}\right\rangle\left\langle\psi_{1} \mathrm{I} \psi_{2}\right\rangle\left\langle\psi_{2} \psi_{0}\right\rangle
$$

is gauge invariant. This is transparent since $\varphi_{\boldsymbol{B}}$ $=\arg \left(\operatorname{tr} \rho_{0} \rho_{1} \rho_{2}\right)$, where $\rho_{j}$ is the density operator for $\left|\psi_{j}\right\rangle$. It turns out that $\varphi_{B}$ is simply related to the geometric phase $\varphi_{g}$ associated with the geodesic triangle $C=\rho_{0} \rho_{1} \rho_{2}$ in ray space.

The importance of geodesies in the context of geometric phase is evident from Pancharatnam's work [10]. Noting that two states $\left|\psi_{j}\right\rangle,\left|\psi_{k}\right\rangle$ are in phase if the inner product $\left\langle\psi_{j} \mid \psi_{k}\right\rangle$ is real positive, along a geodesic arc we can choose states to be in phase with one another. In other words being in phase is an equivalence relation on a geodesic arc. Given a geodesic arc in the ray space determined by two points $P j, p k$ first choose states $\left|\psi_{j}\right\rangle$, $\left|\psi_{k}\right\rangle$ which project, respectively, onto $\rho_{j}, \rho_{k}$, so that $\left\langle\psi_{j} \psi_{k}\right\rangle=\cos \theta$, with $0<\theta<\pi / 2$. Then the continuous family of states

$$
|\psi(s)\rangle=\left[\sin (\theta-s)\left|\psi_{j}\right\rangle+\sin s\left|\psi_{k}\right\rangle\right] / \sin \theta,
$$

for $0 \leq s \leq \theta$, constitutes normalized states along this geodesic, and these states are manifestly in phase with one another.

Geodesies are important also from the point of view of geometric phase experiments, for given a geodesic arc we can always find a constant Hamiltonian which will transport the state along the geodesic with zero-dynamical phase at every instant. Thus, given an $n$-sided geodesic polygon we can find a piecewise constant ( $n$ pieces) Hamiltonian which will transport the state along the polygon in that manner. Inspection of some of the geometric phase experiments [3-5] should convince the reader that 
this fact has indeed played a crucial role in these experiments.

Now we bring out the relationship between the Bargmann phase $\varphi_{B}$ and Berry's geometric phase $\varphi_{g}$. Given $\rho_{0}, \rho_{1}, \rho_{2}$ consider the geodesic triangle $C=\rho_{0} \rho_{1} \rho_{2}$ in the projective space $\mathcal{P}$ of unit rays. The space of normalized state vectors form a $\mathcal{U}(1)$ bundle $\mathcal{N}$ over $\mathcal{P}$. We can lift the closed circuit $C$ in $f$ to a (generally open) curve $G$ in $\mathcal{N}$ as follows. Choose any $\left|\psi_{0}\right\rangle$ in $\mathcal{N}$ atop (i.e., projecting onto) $\rho_{0} ;$ then $\left|\psi_{1}^{\prime}\right\rangle,\left|\psi_{2}^{\prime}\right\rangle,\left|\psi_{0}^{\prime}\right\rangle$, respectively, atop $\rho_{1}, \rho_{2}, \rho_{0}$, respectively, in phase with $\left.\left.\left|\psi_{0}\right\rangle, \mathrm{I} \psi_{1}^{\prime}\right\rangle, \mathrm{I} \psi_{2}^{\prime}\right\rangle$. To complete the lift $G$ of $\mathrm{C}$, construct geodesic arcs in $\mathcal{N}$ as in (2) from $\left|\psi_{0}\right\rangle$ to $\left|\psi_{1}^{\prime}\right\rangle,\left|\psi_{1}^{\prime}\right\rangle$ to $\left|\psi_{2}^{\prime}\right\rangle$, and $\left|\psi_{2}^{\prime}\right\rangle$ to $\left|\psi_{0}^{\prime}\right\rangle$. It is clear that $G$ is a horizontal lift of $\mathrm{C}$, and the dynamical phase along $G$ is zero. Hence the anholonomy $\arg \left\langle\psi_{0} \mid \psi_{0}^{\prime}\right\rangle$ necessarily equals the geometric phase $\varphi_{g}$ associated with the geodesic triangle $C$. Further, $\left\langle\psi_{0} \mid \psi_{1}^{i}\right\rangle,\left\langle\psi_{1}^{\prime} \mid \psi_{2}^{\prime}\right\rangle$, and $\left\langle\psi_{2}^{\prime} \mid \psi_{0}^{\prime}\right\rangle$ are real positive by construction, and $\left|\psi_{0}\right\rangle$ equals $\left|\psi_{0}^{\prime}\right\rangle\left\langle\psi_{0}^{\prime} \mid \psi_{0}\right\rangle$. Using these facts, and the gauge freedom in (1), we deduce

$$
\begin{aligned}
\varphi_{B} & =\arg \left\langle\psi_{0} \mid \psi_{1}^{\prime}\right\rangle\left\langle\psi_{1}^{\prime} \mid \psi_{2}^{\prime}\right\rangle\left\langle\psi_{2}^{\prime} \mid \psi_{0}\right\rangle \\
& =\arg \left\langle\psi_{0}^{\prime} \mid \psi_{0}\right\rangle-\varphi_{g}{ }^{\bullet}
\end{aligned}
$$

We have thus shown that the gauge invariant Bargmann phase $\varphi_{B}$ is simply the negative of the geometric phase $\varphi_{g}$ associated with the geodesic triangle.

As a generalization of the three-vertex Bargmann invariant $\varphi_{B}(3)$ in (1), we can consider the $n$-vertex gauge invariant expression

$$
\varphi_{B}(n)=\arg \left\langle\psi_{0} \mid \psi_{1}\right\rangle\left\langle\psi_{1}|\bullet \cdot| \psi_{n-1}\right\rangle\left\langle\psi_{n-1} \mid \psi_{0}\right\rangle
$$

We can associate with $\varphi_{B}(n)$ an $n$-sided polygon in the ray space, and it is clear that our construction leading to (3) simply generalizes to this case to prove that $\varphi_{B}(n)$ equals the negative of the geometric phase $\varphi_{g}$ associated with this $\boldsymbol{n}$-sided polygon.

It can be shown that the role of geodesies in the above can also be played by geodesies on suitable submanifolds in the ray space, even though these are not described by (2). The cases analyzed hereafter are of this more general kind. As an elementary illustration, consider the oscillator coherent states labeled by the usual complex parameter $a$. Without loss of generality one of the three states can be chosen to be $|0\rangle=|v a c\rangle$. Let the other two states be $\left|\alpha_{1}\right\rangle,\left|\alpha_{2}\right\rangle$. Noting that, with the usual choice of phase, every coherent state is in phase with |0) and that $\arg \left\langle\alpha_{j} \mid \alpha_{k}\right\rangle \operatorname{Im}\left(\alpha_{j}^{*} \alpha_{k}\right)$, we have

$$
\varphi_{B}=\arg \left\langle 0 \mid \alpha_{1}\right\rangle\left\langle\alpha_{1} \mid \alpha_{2}\right\rangle\left\langle\alpha_{2} \mid 0\right\rangle=\operatorname{Im}\left(\alpha_{1}^{*} \alpha_{2}\right) .
$$

Geodesies in the a plane are straight lines, and we see that $\varphi_{B}$ equals twice the area of the geodesic triangle in the $a$ plane. In view of the known result [11] for $\varphi_{g}$ for coherent states, this is consistent with $\varphi_{B}--\varphi_{g}$.

For the purpose of understanding the geometry of the Güoy effect we now turn to one-dimensional Gaussian states with zero mean value for the annihilation operator $a=(q+i p) / \sqrt{2}$. Such states are characterized by a complex width parameter $\xi$ in the lower half complex plane, with normalized wave function $\psi(q ; \xi)=\langle q \mid \xi\rangle$ given by

$$
\psi(q ; \xi)=\pi^{-1 / 4}\left(\operatorname{Im} \xi^{-1}\right)^{1 / 4} \exp \left(i q^{2 / 2 \xi}\right) .
$$

It is the normalizability of $\psi(q ; \xi)$ that restricts $\xi=\xi_{1}$ $+i \xi_{2}$ to the half plane $\xi_{2}<0$. Clearly, the ground state $|\mathrm{vac}\rangle$ now corresponds to $\xi=-i$, and all other states are squeezed Gaussian states. Since $|\xi\rangle$ is annihilated by the unique squeezed boson operator $(q-\xi p)$ / $[2 \operatorname{Im}(-\xi)]^{-2}$, the unitary operators $\exp [-i r(q p+p q) /$ 4] and $\exp \left[-i s p^{2} / 2\right]$ take $|\xi\rangle$, respectively, to $|\xi \exp (r)\rangle$, $\mid \xi+s)$. Thus, $|\xi\rangle$ and $|-i\rangle$ are related as follows, modulo a phase which can be fixed by geometric considerations:

$$
|\xi\rangle=\exp \left(-i \xi_{1} p^{2} / 2\right) \exp \left[-i \ln \left(-\xi_{2}\right)(q p+p q) / 4\right]|-i\rangle \text {. }
$$

Given a closed circuit $\partial \Sigma$, enclosing a region $£$ in the half plane, the associated geometric phase is [1]

$$
\varphi_{g}=i \int_{\partial \Sigma}\left[\left\langle\xi\left|\frac{\partial}{\partial \xi_{1}}\right| \xi\right\rangle d \xi_{1}+\left\langle\xi\left|\frac{\partial}{\partial \xi_{2}}\right| \xi\right\rangle d \xi_{2}\right] \text {. }
$$

Making use of (5) we see that the second term in the integrand is zero, being the expectation value of $\left(\xi_{2}\right)^{-1}(q p+p q) / 4$ in the vacuum state $|-i\rangle$, while the first is the vacuum expectation value of $\left(-\xi_{2}\right)^{-1} p^{2} / 2$ Thus we have

$$
\psi_{g}=-\frac{1}{4} \int_{\partial \Sigma} \frac{d \xi_{1}}{\xi_{2}}=-\frac{1}{A} \iint_{\Sigma} \frac{d \xi_{1} d \xi_{2}}{\left(\xi_{2}\right)^{2}} .
$$

This formula exposes the Lobachevskian hyperbolic metric [12] $d l^{2}=\left(d \xi^{2} d \xi_{2}^{2}\right) / \xi_{2}^{2}$ underlying the manifold of Gaussian states, and we see that the geometric phase is the negative of one-fourth the Lobachevskian area of $\Sigma$, counted positive for anticlockwise traversal of the circuit.

Now consider a generic situation where the phase of any state $|\psi\rangle$ is measured by projecting it onto a fixed reference state $\left|\psi_{R}\right\rangle$. That is, we measure $\arg \left\langle\psi_{R} \mid \psi\right\rangle$, the phase difference in the Pancharatnam sense between $|\psi\rangle$ and $\left|\psi_{R}\right\rangle$. And consider $s$ evolution under a constant Hermitian Hamiltonian $H$. Let $\left|\psi_{E}\right\rangle$ be an eigenstate of $H$ with eigenvalue zero (arranged by adding, if necessary, a real constant to $H)$. Let a state $|\psi\rangle$ evolve from $\left|\psi\left(s_{1}\right)\right\rangle$ to $\left|\psi\left(s_{2}\right)\right\rangle$. Let $\varphi\left(s_{j}\right)=\arg \left\langle\psi_{R}\right| \psi\left(s_{j}\right)$ be the phases of these states. Consider the four-vertex Bargmann invariant

$\varphi_{B}(4)=\arg \left\langle\psi_{R} \mid \psi(s \mid)\right\rangle\left\langle\psi(s \backslash) \mid \psi_{E}\right\rangle\left\langle\psi_{E} \mid \psi\left(s_{2}\right)\right\rangle\left\langle\psi\left(s_{2}\right) \mid \psi_{R}\right\rangle$.

Now $\left.H \backslash \psi_{E}\right\rangle=0$ implies

$$
\left\langle\psi_{E} \mid \psi\left(s_{1}\right)\right\rangle=\left\langle\psi_{E} \mid \psi\left(s_{2}\right)\right\rangle .
$$

Hence, 


$$
\varphi_{B}(4)=-\left[\varphi\left(s_{2}\right)-\varphi\left(s_{1}\right)\right] .
$$

Combining this result with our earlier result $\varphi_{B}--\varphi_{g}$, we arrive at the following.

Theorem: If a state evolves as $|\psi(s)\rangle$ under a constant Hamiltonian $\boldsymbol{H}$, and if its phase at any $s$ is measured with reference to a fixed state $\left|\psi_{R}\right\rangle$, the phase change between $5=s_{1}$ and $s=s_{2}$ equals the geometric phase associated with the geodesic quadrangle $\rho_{R} \rho\left(s_{1}\right) \rho_{E} \rho\left(s_{2}\right)$, where $P E$ corresponds to $\left|\psi_{E}\right\rangle$ with $H\left|\psi_{E}\right\rangle=0$.

It can be seen that the Gu'oy effect belongs precisely to this situation. Let us first consider the one-dimensional case. Calling $x=q$ and $p_{x}=p=-i \mathrm{~d} / \partial q$, the $s$ evolution is generated by the constant Hamiltonian $p^{2} / 2$. The limit of $\left|\xi=0+i \xi_{2}\right\rangle$ in (4) as $\xi_{2} \rightarrow-\infty$ is a real Gaussian with arbitrarily large width (i.e., a constant function in $q$ ); this acts as $\left|\psi_{E}\right\rangle$. Similarly, the limit of $\left|\xi=0+i \xi_{2}\right\rangle$ as $f a-^{\prime} 0$ (from below) is a real Gaussian with arbitrarily small width and hence is a real multiple of the Dirac function $\delta(q)$. This acts as $\left|\psi_{R}\right\rangle$. Finally, the Gu'oy phase $\varphi_{G}(s)$ of the state $|\xi(s)\rangle$ with wave function $\psi(q ; \xi(s))$ is indeed measured by projecting $|\xi(s)\rangle$ onto this $\left|\psi_{R}\right\rangle$ :

$$
\varphi_{G}(s)=\arg \psi(0 ; \xi(s))=\arg \left\langle\psi_{R} \mid \xi(s)\right\rangle .
$$

As already noted, under the constant Hamiltonian $p^{2} / 2$ an initial Gaussian state $\left|\xi_{0}\right\rangle$ evolves as $|\xi(s)\rangle=\left|\xi_{0}+s\right\rangle$, up to a phase. This motion of $\xi(s)$ is parallel to the real axis in the half plane, with a constant imaginary part so there exists a value of $s$ for which $\xi(s)$ is purely imaginary and the width of the wave function is a minimum (waist). We can thus choose without loss of generality (i.e., by shifting the origin of $s$ ) the initial $\xi_{0}$ to be imaginary. That is we take $\xi_{0}=-i w^{2}$, where $w$ is the waist size.

We show in Fig. 1 (a) the half plane constituting our manifold of Gaussian states, and also the states $\rho_{R}, \rho_{\xi\left(s_{1}\right)}$, $P E$, and $\rho_{\xi\left(s_{2}\right)}$. To draw the geodesic quadrangle through these states note that geodesies of the Lobachevskian geometry are circles centered on the real axis, and straight lines normal to this axis [12]. Thus our quadrangle consists of the circular arcs $R \xi\left(s_{1}\right)$ and $\xi\left(s_{2}\right) R$, and the straight lines $\xi\left(s_{1}\right) E$ and $\boldsymbol{E} \xi\left(s_{2}\right)$. Also in this hyperbolic geometry the area of a quadrangle equals the angle deficiency given by $2 \pi$ minus the sum of the interior angles [12]. The angles at $E$ and $R$ are zero. For the other two angles $\alpha_{1}, \alpha_{2}$, note that $\alpha_{j}=2\left(\pi / 2-\theta_{j}\right)$. Thus, the angle deficiency equals $2\left(\theta_{1}+\theta_{2}\right)$ and hence from (6) the geometric phase $\varphi_{g}$ equals $-\left(9 \backslash+\theta_{2}\right) / 2$. From Fig. 1 (a) we have $\tan \theta_{j}=\left|s_{j}\right| / w^{2}$. Thus in view of our main theorem, and noting that $\varphi(s)$ in the generic case of the theorem becomes the Güoy phase $\varphi_{G}(s)$ in the present case, we have our final result

$$
\begin{aligned}
\varphi_{g} & =\varphi_{G}\left(s_{2}\right)-\varphi_{G}\left(s_{1}\right) \\
& =-\frac{-}{2}\left[\arctan \left(s_{2} / w^{2}\right)-\arctan \left(s_{1} / w^{2}\right)\right] .
\end{aligned}
$$

We have thus derived the well-known result [8] $\varphi_{G}(s)$
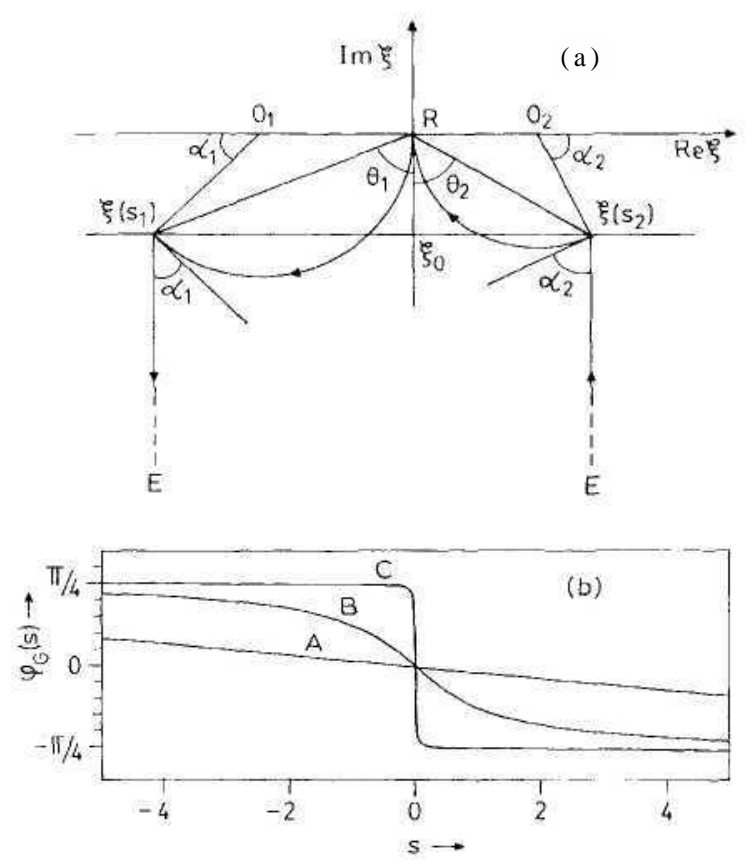

FIG. 1. (a) Showing the Lobachevskian geometry of the Gu'oy effect determined by the geodesic quadrangle $R \xi\left(s_{1}\right) E \xi\left(s_{2}\right)$. The arcs $R \xi\left(s_{1}\right), \xi\left(s_{2}\right) R$ are centered, respectively, at $\mathrm{O}_{1}, \mathrm{O}_{2}$, (b) The $s$ evolution of the Gu'oy phase; $A, B$, and $C$ correspond to $w=5,1$, and 0.1 , respectively.

$=-\overline{2} \arctan \left(s / w^{2}\right)$ based entirely on Berry-Bargmann geometric phase considerations. In Fig. 1 (b) we show $\varphi_{G}(s)$ for various values of the waist size $w$. The magnitude of the Gu'oy phase jump is $\pi / 2$ precisely because the area of geodesic quadrangles in the Lobachevskian geometry is bounded by $2 \pi$, and that the Giioy phase change becomes abrubt as $w \rightarrow 0$ (i.e., as $\xi_{0}$ approaches the real axis) because the area is concentrated near the real axis.

For the Hermite-Gaussian beam of order $n$, the role of $|v a c\rangle$ is played by the $n$th eigenstate $|n\rangle$. Thus $\left(p^{2}\right)$ leading to (6), and hence the Gu'oy phase, get scaled by $(2 n+1)$. For two-dimensional beams, the $x$ and $y$ Giioy phases add.

Bargmann originally introduced his gauge invariant $\varphi_{B}$ for the express purpose of distinguishing between unitary and antiunitary symmetry operators through the behavior of $\varphi_{B}$ under them. In a recent experiment Tompkin et al. [13] studied the behavior of geometric phase in the presence of a (antiunitary) phase conjugate mirror. It is interesting to note that their experiment fully verifies Bargmann's original ideas, even if these authors were apparently unaware of his work.

The geometric phases associated with the rotation group $\mathrm{SO}(3)$ and its double cover $\mathrm{SU}(2)$ have been studied in several experiments [3-5]. These equal, respectively, the solid angle and half the solid angle on the unit 
sphere $S^{2}$. The geometric phase associated with the Lorentz group $\mathrm{SO}(2,1)$ and its double cover $\mathrm{Sp}(2, R)$ - SU $(1,1)$ has also been studied [14]. Our unitary evolutions generated by Hamiltonians quadratic in $q, p$ constitute not $\operatorname{Sp}(2, R) \sim \operatorname{SU}(1,1)$ but the metaplectic group [15] $M p(2)$, which is a double cover of $\operatorname{Sp}(2, R)$, and henceforth cover of $\mathrm{SO}(2,1)$. And this explains the $\frac{\overline{4}}{4}$ factor in the geometric phase formula (6). We can thus summarize by saying that the century-old Güoy phase, given by one-fourth Lobachevskian area, and encountered in routine laser optics, is the Berry phase associated with the metaplectic group.

The Giioy effect for squeezed light [16] is also governed by our formalism. Since "free" evolution in this case is generated by $\left(q^{2}+p^{2}\right) / 2$, the role of $\left|\psi_{E}\right\rangle$ is played by $|-i\rangle$.

One of us (R.S.) would like to thank M. V. Berry for a discussion on these matters.

[1] M. V. Berry, Proc. R. Soc. London A 392, 45 (1984).

[2] B. Simon, Phys. Rev. Lett. 51, 2167 (1983); F. Wilczek and A. Zee, ibid. 52, 2111 (1984); Y. Aharonov and J. Anandan, ibid. 58, 1593 (1987).

[3] A. Tomita and R. Y. Chiao, Phys. Rev. Lett. 57, 937 (1986); M. Segev, R. Solomon, and A. Yariv, ibid. 69,
590 (1992).

[4] R. Bhandari and J. Samuel, Phys. Rev. Lett. 60, 1210 (1988); P. G. Kwiat and R. Y. Chiao, ibid. 66, 588 (1991).

[5] T. H. Chyba, L. J. Wang, L. Mandel, and R. Simon, Opt. Lett. 13, 562 (1988); R. Simon, H. J. Kimble, and E. C. G. Sudarshan, Phys. Rev. Lett. 61, 19 (1988).

[6] V. Bargmann, J. Math. Phys. 5, 862 (1964).

[7] G. Güoy, C. R. Acad. Sci. Paris 110, 125 (1890).

[8] A. E. Siegman, Lasers (Oxford Univ. Press, Oxford, 1986), Chap. 17.

[9] D. Gloge and D. Marcuse, J. Opt. Soc. Am. 59, 1629 (1969).

[10] S. Pancharatnam, Proc. Ind. Acad. Sci. A 44, 247 (1956).

[1I] S. Chaturvedi, M. S. Sriram, and V. Srinivasan, J. Phys. A 20, L1071 (1987); R. Simon and N. Kumar, ibid. 21, 1725 (1988).

[12] M. Berger, Geometry II (Springer-Verlag, Berlin, 1987), Chap. 19.

[13] W. R. Tompkin, M. S. Malcuit, W. R. Boyd, and R. Y. Chiao, J. Opt. Soc. Am. B 7, 230 (1990).

[14] T. F. Jordan and R. Y. Chiao, Phys. Lett. A 132, 77 (1988); G. S. Agarwal and R. Simon, Phys. Rev. A 42, 6924 (1990); M. Kitano and T. Yabuzaki, Phys. Lett. A 142, 321 (1989); G. S. Agarwal, Opt. Commun. 82, 213 (1991).

[15] H. Bacry and M. Cadilhac, Phys. Rev. A 23, 2533 (1981); R. Simon, Curr. Sci. 59, 1168 (1990).

[16] R. Simon and N. Mukunda, Opt. Commun. (to be published). 